konstanten Wert $F_{0}-n_{\mathrm{K}}$ ausläuft, steigen unsere Dispersionskurven weit über diesen Wert hinaus und erreichen zwischen der K-Kante und den L-Kanten ein Maximum, das beim Tellur höher als $F_{0}$ liegt und beim Wolfram beinahe bis an diese Größe heranreicht. Es scheint daher nicht möglich, die Zahl der K-Dispersionselektronen in der bisherigen einfachen Weise experimentell zu bestimmen, wie dies ohne Kenntnis des Einflusses der anomalen L-Dispersion versucht worden ist ${ }^{5}$. Während für Tellur noch keine experimentellen Werte der Dispersion vorliegen, haben Brentano und Baxter ${ }^{6}$ bereits vor längerer Zeit Dispersionsmessungen an Wolfram in der Nähe der L-Kanten ausgeführt. Diese (durch $x \times$ in das Wolfram-Diagramm eingetragen) bestätigen die von uns gefundene Überhöhung des Dispersionsverlaufs zwischen der K- und den L-Kanten. Die beiden Meßpunkte in der Nähe der $\mathrm{L}_{\mathrm{I}}$-Kante liegen allerdings etwas zu tief, während die beiden Meßwerte auf der langwelligen Seite der L-Kanten

${ }^{5}$ Vgl. R. W. James, The Optical Principles of the Diffraction of X-Rays, London 1950. wieder befriedigend mit unserer Kurve übereinstimmen. Man beachte, daß in diesen langwelligen Spektralbereich bereits die anomale Dispersion der M-Schale einwirkt, die in der vorliegenden Untersuchung nur teilweise berücksichtigt werden konnte. Die bezüglich der gesamten anomalen M-Dispersion korrigierte Dispersionskurve würde analog den oben besprochenen Verhältnissen an der K-Kante nach höheren $F$-Werten hin verschoben werden, also zugunsten der beiden auf der langwelligen Seite der L-Kanten gefundenen experimentellen Werte.

Eine ausführliche Mitteilung mit Darlegung des Rechenganges erfolgt demnächst in der ,Zeitschrift für Physik".

Herrn Prof. H. Hönl danken wir an dieser Stelle für die Anregung zu dieser Arbeit und sein förderndes Interesse. Für die großzügige Unterstützung der Arbeit durch die Deutsche Forschungsgemeinschaft sei dieser ebenfalls bestens gedankt.

6 J. Brentano u. A. Baxter, Z. Physik 89, 720 [1934].

\title{
Elektronenzustände kubischer Kristalle in Brillouinscher Näherung*
}

\author{
Von J. Homilius \\ Aus dem Institut für theoretische Physik der Universität Münster i. Westf.
}

(Z. Naturforschg. 8a, 432-441 [1953]; eingegangen am 29. Mai 1953)

\begin{abstract}
Eigenfunktionen und Energieflächen für die kubischen Gitter $\Gamma_{c}, \Gamma_{c}^{\prime}$ und $\Gamma_{c}{ }^{\prime \prime}$ werden in erster Brillouinscher Näherung behandelt und insbesondere für das kubische Flächengitter näher untersucht. Es werden die Energieaufspaltungen in den Umgebungen der ersten sechs Resonanzstellen berechnet und Energiebänder längs charakteristischer Geraden auf den Begrenzungsflächen der ersten Brillouin-Zonen graphisch dargestellt.
\end{abstract}

$Z^{n}$ ur Berechnung der inneren Feldemission in Einkristallen nach dem Houstonschen Ansatz ${ }^{1,2}$ ist die Kenntnis der Elektroneneigenfunktionen im Kristall und besonders der dazugehörigen Energiewerte in Abhängigkeit von den Impulsen bzw. Wellenzahlen nötig, und zwar kommt es besonders auf die Verhältnisse an den Bandgrenzen an. Aus den vorhandenen Rechnungen lassen sich meist nur der Energieverlauf längs Geraden, welche innerhalb der Zonen liegen, und die Wellenfunktion fast stets nur

* Erster Teilauszug aus der Dissertation, Münster i. Westf.

1 W. V. Houston, Physic. Rev. 57, 184 [1940]. für bestimmte Punkte des Wellenzahlraumes entnehmen. Eine für den ganzen Wellenzahlraum gültige Lösung wurde von Slater ${ }^{3}$ angegeben, doch ist sein Potentialansatz zu speziell, da er nur Fourier-Koeffizienten $v_{100}$ enthält; die resultierenden Funktionen sind für unsere Zwecke zu schwer zu handhaben. Um Wellenfunktionen und Energieflächen in einer möglichst einfachen analytischen Gestalt zu gewinnen, welche die etwas komplizierten Operationen der Houstonschen Theorie zuläßt,

\footnotetext{
2 J. Homilius, Z. Naturforschg. 7 a, 290 [1952].

${ }^{3}$ J. C. Slater, Physic. Rev. 87, 807 [1952].
} 
wird im folgenden das Problem in der Näherung von freien Elektronen behandelt. Dabei müssen wir allerdings, um für den ganzen Wellenzahlraum handliche Funktionen zu erhalten, auf den Grad von Exaktheit verzichten, welcher mit verschiedenen anderen Methoden für spezielle Wellenzahlen erreicht wurde. Doch wird die hier angewandte erste Brillouinsche Näherung für spezielle Fälle eine befriedigende Beschreibung darstellen, wie Fues und Statz ${ }^{4}$ gezeigt haben.

Wir beschränken uns auf kubische Kristalle vom Typ $\Gamma_{c}, \Gamma_{c}^{\prime}$ und $\Gamma_{c}{ }_{c}^{\prime \prime}$ und nennen $a$ die Seitenlänge des elementaren Würfels. Um die Formeln möglichst zu vereinfachen, messen wir die Länge in Einheiten $a / \pi$, die Wellenzahl $\mathfrak{f}$ in Einheiten $\pi / a$ und die Energie in Einheiten

$$
P=\frac{\hbar^{2}}{m} \cdot \frac{\pi^{2}}{a^{2}}=8,358 \cdot\left(\frac{3 \AA}{a}\right)^{2} \mathrm{eV}
$$

( $m=$ Elektronenmasse). Die Schrödinger-Gleichung nimmt dann folgende Gestalt an

$$
(H-E(\mathfrak{f})) \Psi(\mathfrak{r}, \mathfrak{l})=0 .
$$

Darin ist $H=H^{0}+V(\mathfrak{r})$ mit

$$
\begin{array}{r}
H^{0}=-\frac{A}{2}-v_{0} \quad \text { und } V(\mathfrak{r})=-\sum_{\mathfrak{g} \neq 0} v_{\mathfrak{g}} e^{2 i \mathrm{~g} \cdot \mathfrak{r}}, \\
\mathfrak{g}=\sum_{l=1}^{3} g^{l} \mathfrak{b}_{l}\left(g^{l} \text { ganz }\right) .
\end{array}
$$

Bei diesem Ansatz des periodischen Potentials $V(\mathfrak{r})$ des Gitters werden für einen Isolator die niedrigst indizierten Koeffizienten $v_{\mathfrak{g}}$ positiv. Die Blochsche Wellenfunktion im Energieband sei

$$
\Psi_{r}(\mathfrak{r}, \mathfrak{f})=e^{i \mathfrak{f} \cdot \mathfrak{r}} \chi_{r}(\mathfrak{r}, \mathfrak{t})
$$

mit $\mathfrak{f}$ als nichtreduziertem Wellenzahlvektor. $\chi_{r}$ sei im Elementarvolumen $\Omega_{0}=a^{3}$, bzw. in unseren Einheiten $=\pi^{3}$ normiert.

Die Abhängigkeit der Energie von der Wellenzahl, welche beim freien Elektron parabolisch ist, wird durch die Braggschen Reflexionen längs der sog. Brillouin-Ebenen $\left\{m^{1} m^{2} m^{3}\right\}$

$$
m^{1} k_{x}+m^{2} k_{y}+m^{3} k_{z}=|\mathrm{m}|^{2} \quad\left(m^{i} \text { ganz }\right)
$$

aufgespalten (Abb. 1). Maßgeblich für die Größe der Aufspaltung ist der zugehörige Fourier-Koeffizient $v_{\mathfrak{m}}$ des Potentials. Wir betrachten speziell die Fourier-Komponente

$$
V_{m x} \equiv-v_{m 00}\left(e^{2 i m x}+e^{-2 i m x}\right) .
$$

4 E. Fues u. H.Statz, Z. Naturforschg. 7a, 2 [1952].
Sie bewirkt eine Aufspaltung in der Umgebung der Ebene $\{m 00\}$; wir greifen als ,Resonanzstelle“ (= Punkt im Wellenzahlraum mit mindestens einfacher Entartung) den Punkt $\mathfrak{f}_{0}^{(0)}=(m 00)$ heraus und schreiben den allgemeinen Vektor $\mathfrak{f}$ mit $\mathfrak{f}_{1}^{(0)}=\left(\Delta_{x}^{(0)}\right.$, $\Delta_{y}^{(0)}, \Delta_{z}^{(0)}$ ) in der Form:

$$
\mathfrak{f}=\mathfrak{f}_{0}^{(0)}+\mathfrak{f}_{1}^{(0)} .
$$

Bei $\mathfrak{f}_{0}^{(0)}$ sind die Wellen mit den Wellenzahlvektoren $\mathfrak{f}$ und $\mathfrak{f}-2 m \mathfrak{b}_{1}$ entartet. Die Energieaufspaltung

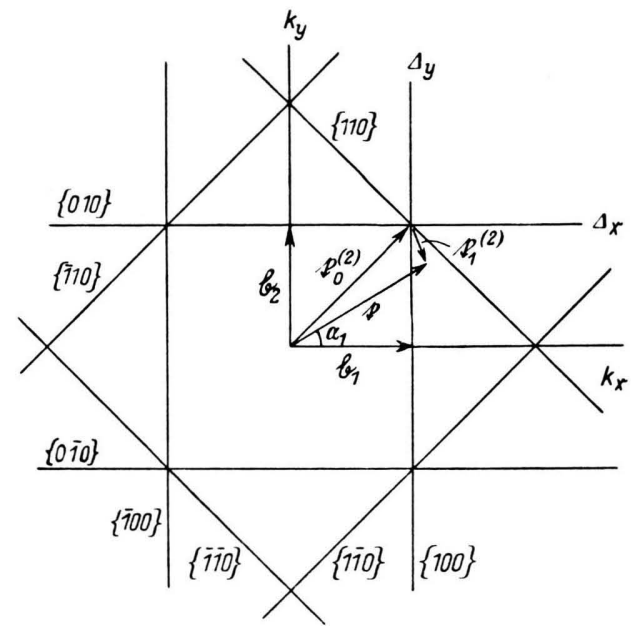

Abb. 1. Erste B.-Zone des Gitters $\Gamma_{c}$ in der Ebene $k_{z}=0$ mit den B.-Ebenen vom Typ $\{100\}$ und $\{110\}$ und der Vektorzerlegung $\mathfrak{t}=\mathfrak{f}_{i j}{ }^{(2)}+\mathfrak{f}_{1}{ }^{(2)}$.

ist bekanntlich ${ }^{5}$

$E_{1}=\bar{E}^{(0)} \mp \varepsilon_{m x}, \quad \varepsilon_{m x} \equiv+\sqrt{m^{2} \Delta^{2}{ }_{x}+v^{2}{ }_{m 00}}$

mit der mittleren Energie

$$
\bar{E}^{(0)}=\bar{E}\left(\mathfrak{f}_{0}^{(0)}, \mathfrak{f}_{1}^{(0)}\right) \equiv \frac{\mathfrak{f}_{0}\left({ }^{(0) 2} 2\right.}{2}+\frac{\mathfrak{f}_{1}(0) 2}{2}-v_{0} .
$$

Die zugehörigen Funktionen haben die Gestalt

$$
\chi_{\frac{1}{2}}=\frac{1}{\sqrt{\pi^{3}}} \chi_{0}^{u}(m x)
$$

mit

$$
\begin{aligned}
\sqrt{2} \chi_{u}(m x) & =\sqrt{1+\gamma_{m x}}+\sqrt{1-\gamma_{m x}} e^{-2 i m x}, \\
\sqrt{2} \chi_{0}(m x) & =-\sqrt{1-\gamma_{m x}}+\sqrt{1+\gamma_{m x}} e^{-2 i m x}, \\
\gamma_{m x} & \equiv \frac{m \Delta_{x}}{\varepsilon_{m x}} .
\end{aligned}
$$

Die Teilfunktionen (11) erster Brillouinscher Näherung bilden die Grundlage der folgenden Rechnungen, welche successiv verschiedene höhere Braggsche Reflexionen berücksichtigen, jedoch ebenfalls nur in dem gleichen Grade der Näherung.

5 A. Sommerfeld u. H. Bethe, Handb. d. Physik 24 II, 333, Berlin [1933]. 
Für die späteren Untersuchungen werden die Matrixelemente der Funktionen (11) benötigt:

$$
\begin{gathered}
\left(\chi_{0}, \chi_{u}\right)=0 ;\left(\chi_{0}^{u}, \chi_{0}^{u}\right)=\pi ;\left(\chi_{0}^{u}, \frac{\partial \chi_{0}^{u}}{\partial \Delta_{x}}\right)=0 ; \\
\left(\chi_{u}, \frac{\partial \chi_{0}}{\partial \Delta_{x}}\right)=-\left(\chi_{0}, \frac{\partial \chi_{u}}{\partial \Delta_{x}}\right)=X_{m} \pi ; X_{m} \equiv \frac{m}{2} \frac{v_{m 00}}{\varepsilon^{2} m x} .
\end{gathered}
$$

Das durch die Klammern angedeutete innere Produkt der Funktionen enthält eine eindimensionale Integration über $x$ von 0 bis $\pi$.

\section{Kubisches Gitter $\Gamma_{c}$ in}

\section{zweidimensionaler Betrachtung}

Den Übergang zum ebenen Problem erreichen wir dadurch, daß wir als Resonanzstelle den Vektor $\mathfrak{f}_{0}^{(2)}=(110) *$ herausgreifen (Abb. 1). Da die $\mathfrak{f}_{0}^{(s)}$ Ortsvektoren des Wellenzahlraumes sind, können wir die Endpunkte mit $P_{s}$ bezeichnen. Dann liegt der zu f $\mathfrak{f}_{0}^{(2)}$ gehörige Punkt $\mathrm{P}_{2}$ auf der von den Ebe-

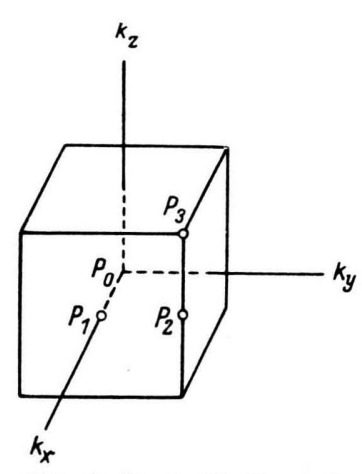

Abb. 2. Erste B.-Zone des Gitters $\Gamma_{c}$ mit den Punkten $P_{0}=(000), P_{1}=(100)$, $P_{2}=(110)$ und $P_{3}=(111)$. nen vom Typ $\{100\}$ begrenzten würfelförmigen B.-Zone, und zwar in der Mitte der Kante zweierBegrenzungsflächen,nämlich der $\{100\}$ - und $\{010\}$ Fläche (Abb. 2). Zu $\mathfrak{f}_{0}^{(2)}$ wählen wir den variablen Vektor $\mathfrak{f}_{1}^{(2)}$ so, daß er sich nur in der Ebene $k_{z}=0$ bewegt; er habe die Gestalt $\mathfrak{f}_{1}^{(2)}=\left(\Delta_{x}, \Delta_{y}, 0\right)$. Bei $\mathfrak{f}_{0}^{(2)}$ sind die folgenden vier Wellen entartet: $\mathfrak{f}, \mathfrak{f}-2 \mathfrak{b}_{1}$, $\mathfrak{f}-2 \mathfrak{b}_{2}, \mathfrak{f}-2\left(\mathfrak{b}_{1}+\mathfrak{b}_{2}\right)$. Die Vektoren $\mathfrak{b}_{1}, \mathfrak{b}_{2}, \mathfrak{b}_{3}$ sollen dabei, entsprechend Abb. 1, die Grundvektoren des reziproken Gitters sein. Die mittlere Energie $\bar{E}^{(2)}=\bar{E}\left(\mathfrak{f}_{0}^{(2)}, \mathfrak{f}_{1}^{(2)}\right)$ der vier Wellen zeigt als Funktion von $\mathfrak{f}_{1}^{(2)}$ einen parabolischen Verlauf analog zu (9). Die Abweichung der Energie einer einzelnen Welle von der mittleren Energie der vier Wellen ist eine lineare Funktion von $\Delta_{x}$ und $\Delta_{y}$ (Abb. 3). Die Hamilton-Funktion des Schrödinger-Problems (2) setzt sich nun aus $H^{0}$ und $H^{1}$ zusammen:

$$
H^{1}=V_{x}+V_{y}+V_{z}, V_{x_{l}}=-v_{100}\left(e^{2 i x_{l}}+e^{-2 i x_{l}}\right) .
$$

* Mit $\mathfrak{f}_{0}^{(1)}$ werde der Vektor (100) in Tab. 1 und 2 bezeichnet.
Der Störung $H^{0}+H^{1}$ kann man durch den folgenden Produktansatz aus den Funktionen (11) Rechnung tragen:

$$
\begin{aligned}
& \chi_{1}=\frac{1}{\sqrt{\pi^{3}}} \chi_{u}(x) \chi_{u}(y), \Delta_{1}=E_{1}-\bar{E}^{(2)}=-\varepsilon_{x}-\varepsilon_{y} \\
& \chi_{2}=\frac{1}{\sqrt{\pi^{3}}} \chi_{0}(x) \chi_{u}(y), \Delta_{2}=E_{2}-\bar{E}^{(2)}=+\varepsilon_{x}-\varepsilon_{y} \\
& \chi_{3}=\frac{1}{\sqrt{\pi^{3}}} \chi_{u}(x) \chi_{0}(y), \Delta_{3}=E_{3}-\bar{E}^{(2)}=-\varepsilon_{x}+\varepsilon_{y} \\
& \chi_{4}=\frac{1}{\sqrt{\pi^{3}}} \chi_{0}(x) \chi_{0}(y), \Delta_{4}=E_{4}-\bar{E}^{(2)}=+\varepsilon_{x}+\varepsilon_{y}
\end{aligned}
$$

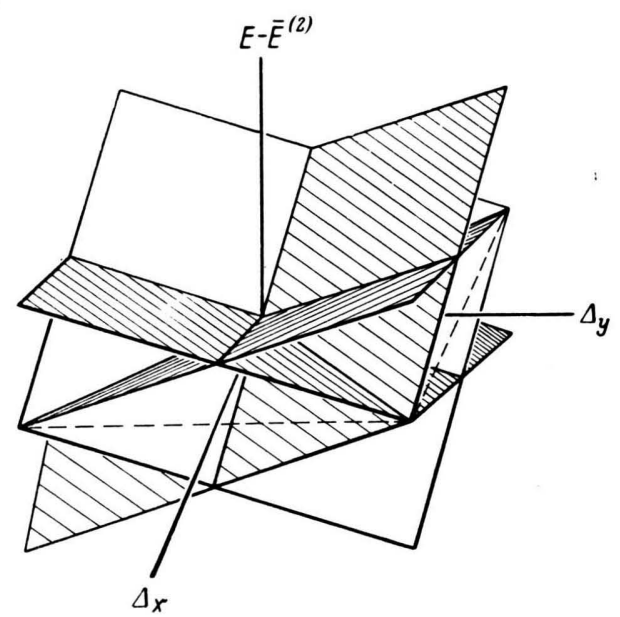

Abb. 3. Abweichungen der Energiewerte von der mittleren Energie $\bar{E}^{(2)}$ für die vier in $\mathrm{P}_{2}$ voll entarteten ebenen Wellen über der Ebene $k_{z}=0$.

Darin sind $\Delta_{r}$ die Abweichungen von der mittleren Energie $\bar{E}^{(2)}$; die Größen $\varepsilon_{x_{l}}\left(x_{2}=x, y, z\right)$ sind durch

$$
\varepsilon_{x_{l}} \equiv+\sqrt{\Delta^{2}{ }_{x_{l}}+v^{2}{ }_{100}}
$$

definiert. Den Verlauf der Energieflächen über $\bar{E}^{(2)}$ in der Umgebung der Resonanzstelle $\mathfrak{f}_{0}^{(2)}$ gibt in diesem Grade der Näherung Abb. $4 \mathrm{a}$ und 4 b. Um auch den Fourier-Koeffizienten $v_{110} \mathrm{zu}$ berücksichtigen, müssen wir die Hamilton-Funktion aus den Teilen $H^{0}, H^{1}$ und $H^{2}$ additiv zusammensetzen mit

$$
\begin{aligned}
& H^{2}=V^{x}+V^{y}+V^{z}, \\
& V^{x_{l}}=-v_{110}\left(e^{2 i x_{l-1}}+e^{-2 i x_{l}-1}\right) \\
& \cdot\left(e^{2 i x_{l+1}}+e^{2 i x_{l+1}}\right) .
\end{aligned}
$$

Für die Eigenfunktionen machen wir den Ansatz

$$
\Psi=e^{i \boldsymbol{i} \cdot \mathrm{r}} \sum_{r=1}^{4} \alpha^{r} \chi_{r}+\varphi .
$$


Für $\varphi$ erhält man folgende inhomogene Diffgl.

$\left(E^{2}-H\right) \varphi=e^{i \ddagger \cdot r} \sum_{r=1}^{4}\left(\Delta_{r}-\lambda+H^{2}\right) \alpha^{r} \chi_{r}$.

Darin sind $\Delta_{r}$ die Abweichungen von der mittleren Energie nach (14) ohne, $\lambda$ die Abweichungen von der mittleren Energie mit Berücksichtigung der Störung
$H^{2}$. In üblicher Weise entsteht durch Orthogonalisierung für die $\alpha^{r}$ das Eigenwertproblem

$\sum_{r=1}^{4}\left[\left(\lambda-\Delta_{r}\right) \delta_{r m}-T_{r m}^{2}\right] \alpha^{r}=0$

$$
(m=1,2,3,4)
$$

mit der Matrix

$$
\left(T_{r m}^{2}\right)=\left(\chi_{r}, V^{z} \chi_{m}\right)=-\left(\begin{array}{cccr}
u & v_{x} & v_{y} & w \\
v_{x} & -u & w & -v_{y} \\
v_{y} & w & -u & -v_{x} \\
w & -v_{x}-v_{x} & u
\end{array}\right),
$$

worin

$$
u=v_{110} \frac{v_{100}^{2}}{\varepsilon_{x} \varepsilon_{y}}, v_{x}=v_{110} \frac{\Delta_{x} v_{100}}{\varepsilon_{x} \varepsilon_{y}}, v_{y}=v_{110} \frac{\Delta_{y} v_{100}}{\varepsilon_{x} \varepsilon_{y}}, w=v_{110} \frac{\Delta_{x} \Delta_{y}}{\varepsilon_{x} \varepsilon_{y}} .
$$

Die Eigenwerte $\lambda_{r}$ bestimmen sich aus der Säkulargleichung von (19)

$$
\begin{gathered}
{\left[(\lambda+u)^{2}-\left(\varepsilon_{x}+\varepsilon_{y}\right)^{2}\right] \cdot\left[(\lambda-u)^{2}-\left(\varepsilon_{x}-\varepsilon_{y}\right)^{2}\right]=R\left(\lambda^{2}\right),} \\
R\left(\lambda^{2}\right) \equiv 2 \lambda^{2}\left(v_{x}{ }^{2}+v_{y}{ }^{2}+w^{2}\right)-2\left(v_{x}{ }^{2}-v_{y}{ }^{2}\right)\left(\varepsilon_{x}{ }^{2}-\varepsilon_{y}{ }^{2}\right)-2 w^{2}\left(\varepsilon_{x}{ }^{2}+\varepsilon_{y}{ }^{2}\right) \\
-2 u^{2}\left(2 w^{2}+v_{x}{ }^{2}+v_{y}{ }^{2}\right)-2 w^{2}\left(v_{x}{ }^{2}+v_{y}{ }^{2}\right)-w^{4}-v_{x}{ }^{4}-v_{y}{ }^{4} .
\end{gathered}
$$

Sie ist als Gleichung vierten Grades in $\lambda$ zwar exakt auflösbar, liefert jedoch die funktionelle Abhängigkeit von $\mathfrak{f}_{1}^{(2)}$ in zu komplizierter Weise, als daß wir sie für unsere weitere Rechnung verwenden könnten. Wir suchen daher Näherungslösungen für kleine Werte der Parameter $v_{110}, \Delta_{x}$ bzw. $\Delta_{y}$ und $\Delta_{x}-\Delta_{y}$. Eine erste Näherung in $v_{110}$ ist sofort angebbar:

$$
\left.\begin{array}{l}
\lambda_{1}=\Delta_{1}-u \\
\lambda_{2}=\Delta_{2}+u \\
\lambda_{3}=\Delta_{3}+u \\
\lambda_{4}=\Delta_{4}-u
\end{array}\right\}+o\left(v^{2}{ }_{110}\right) .
$$

Um höhere Näherungen zu erhalten, führen wir einen iterativen Ansatz durch, und zwar speziell für $\lambda_{1}$ und $\lambda_{4}$

$(\lambda+u)-\left(\varepsilon_{x}+\varepsilon_{y}\right)^{2}=R\left(\lambda^{2}\right) /\left[(\lambda-u)^{2}-\left(\varepsilon_{x}-\varepsilon_{y}\right)^{2}\right]$

(für $\lambda_{2}$ und $\lambda_{3}$ entsprechend), welcher zu folgenden Entwicklungen von $\lambda_{r}$ in den Koeffizienten von $v_{110}$ bis zu Gliedern vierten Grades führt:

Abb. 4. Energieflächen in Einheiten $v_{100}$ über der Ebene $k_{z}=0$ in der Umgebung von $\mathrm{P}_{2}$. Aufgetragen sind die Abweichungen von der mittleren Energie $\bar{E}^{(2)}$. (a) Erste Zone, (b) zweite Zone bei Berücksichtigung von $v_{100}$; (c) erste Zone, (d) zweite Zone, (f) dritte Zone, (e) vierte Zone bei Berücksichtigung von $v_{100}$ und $v_{110}$ $\left(=v_{100} / 2\right)$.

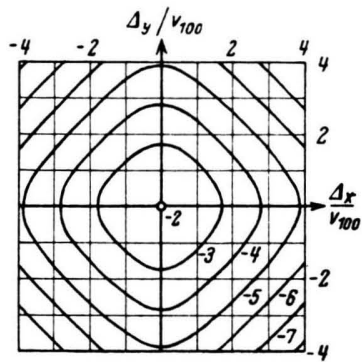

a
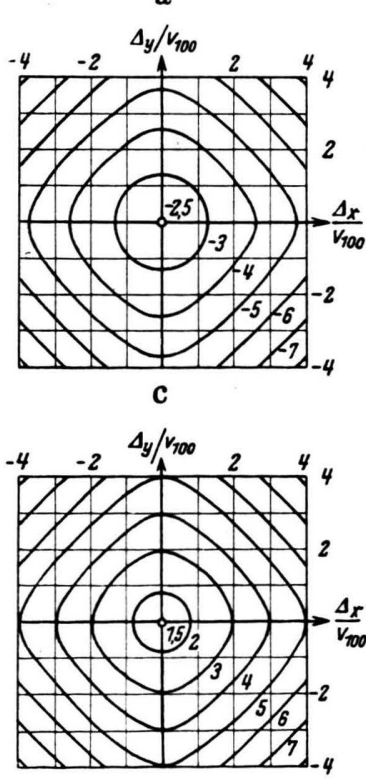

e
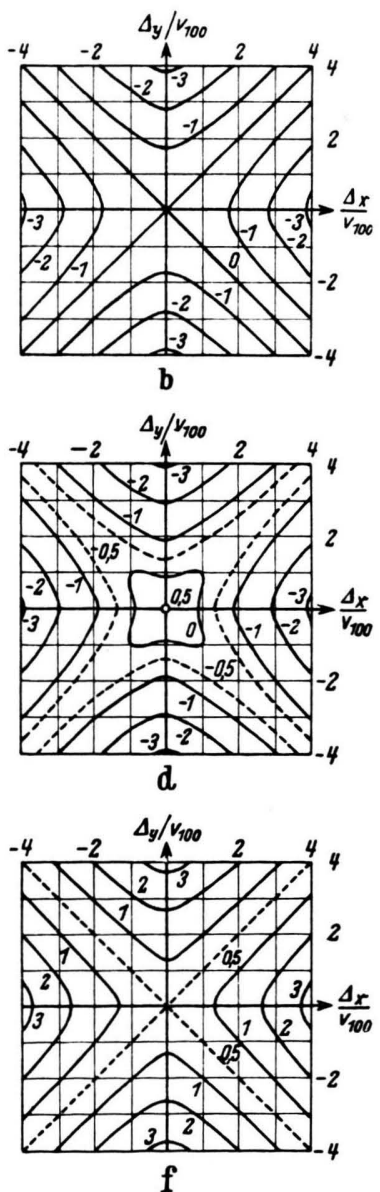
$\lambda_{1}=-u\left(1-\frac{1}{2} \frac{v_{x}{ }^{2}}{\varepsilon_{x}{ }^{2}}-\frac{1}{2} \frac{v_{y}{ }^{2}}{\varepsilon_{y}{ }^{2}}\right) \mp\left[\left(\varepsilon_{x}+\varepsilon_{y}\right)^{2}\right.$

$\left.+w^{2}+\left(1+\frac{\varepsilon_{y}}{\varepsilon_{x}}\right) v_{x}{ }^{2}+\left(1+\frac{\varepsilon_{x}}{\varepsilon_{y}}\right) v_{y}{ }^{2}\right]^{1}+\mathrm{o}\left(v^{4}{ }_{110}\right)$,

$\lambda_{\frac{2}{3}}=u\left(1-\frac{1}{2} \frac{v_{x}{ }^{2}}{\varepsilon_{x}{ }^{2}}-\frac{1}{2} \frac{v_{y}{ }^{2}}{\varepsilon_{y}{ }^{2}}\right) \mp\left[\left(\varepsilon_{x}-\varepsilon_{y}\right)^{2}\right.$

$\left.+w^{2}+\left(1-\frac{\varepsilon_{y}}{\varepsilon_{x}}\right) v_{x}{ }^{2}+\left(1-\frac{\varepsilon_{x}}{\varepsilon_{y}}\right) v_{y}{ }^{2}\right]^{\frac{1}{2}}+o\left(v^{4}{ }_{110}\right)$.

Die aus diesen Gleichungen berechneten Energieflächen über der mittleren Energie sind in Abb. $4 \mathrm{c}$ bis $4 \mathrm{f}$ für einen speziellen Wert des Parameterverhältnisses $v_{110} / v_{100}$ angegeben.

Eine weitere Näherungslösung für kleine $A_{y}$ gewinnen wir sofort aus (22), indem wir dort $v_{y}{ }^{2}$ und $w^{2}$ vernachlässigen. Die Lösungen lauten für $v_{100}$, $v_{110}>0$ :

$$
\begin{aligned}
& \lambda_{\frac{3}{3}}=-v_{100} \mp \sqrt{\Delta^{2}{ }_{x}+\left(v_{100}+v_{110}\right)^{2}+o\left(\Delta^{2}{ }_{y}\right),} \\
& \lambda_{\frac{2}{4}}=v_{100} \mp \sqrt{\Delta^{2}{ }_{x}+\left(v_{100}-v_{110}\right)^{2}}+o\left(\Delta^{2}{ }_{y}\right) .
\end{aligned}
$$

Eine analoge Näherungslösung für kleine $\Delta_{x}$ erhält man durch Vertauschung von $\Delta_{x}$ mit $\Delta_{y}$.

Schließlich bekommen wir aus (25) eine Lösung auf der zu $\Delta_{x}+\Delta_{y}=0$ parallelen Geradenschar, indem wir den Scharparameter $\eta$ durch $\Delta_{x}=\xi-\eta$, $\Delta_{y}=\xi+\eta$ einführen und nach $v_{110}$ und $\eta$ entwickeln. Mit der Abkürzung $\varepsilon^{2}=\xi^{2}+v^{2}{ }_{100}$ ergibt $\operatorname{sich}\left(v_{110}>0\right)$ :

$\lambda_{1}=-v_{110} \frac{v^{2}{ }_{100}}{\varepsilon^{2}}$

$\mp 2 \sqrt{\xi^{2}+v^{2}{ }_{100}+\frac{v^{2}{ }_{100}}{4} \cdot \frac{\xi^{2}\left(\xi^{2}+4 v^{2}{ }_{100}\right)}{\varepsilon^{4}}+O\left(\eta^{2}, v^{3}{ }_{110}\right)}$,

$\lambda_{2}=v_{110} \frac{v^{2}{ }_{100}}{\varepsilon^{2}} \mp v_{110} \frac{\xi^{2}}{\varepsilon^{2}}+\mathrm{o}\left(\eta^{2}, v^{3}{ }_{110}\right)$.

Für $\eta=0$ erhält man aus der Säkulargl. (22) exakt die Gleichung dritten Grades

$$
\begin{array}{r}
\left(\lambda+v_{110}\right)^{3}-2\left(\lambda+v_{110}\right)^{2} v_{110}-4 \varepsilon^{2}\left(\lambda+v_{110}\right) \\
+8 v_{110} v_{100}^{2}=0
\end{array}
$$

und die lineare Gleichung $\lambda-v_{110}=0$. (28) hat die exakte Cardanosche Lösung

$$
\begin{aligned}
\lambda_{1} & =-\frac{v_{110}}{3} \mp 2 \sqrt{p} \cos \frac{\chi}{3}-\frac{2}{3} \sqrt{p} \sin \frac{\chi}{3}, \\
\lambda_{2} & =-\frac{v_{110}}{3}+\frac{4}{\sqrt{3}} \sqrt{p} \sin \frac{\chi}{3}, \\
\chi & =\arcsin x\left(\approx x+\frac{x^{3}}{6}\right), x=\frac{\sqrt{3}}{2} \frac{q}{\sqrt{p^{3}}}, \\
q & =v_{110}\left\{2\left[v^{2}{ }_{100}-\left(\frac{v_{110}}{3}\right)^{2}\right]-\xi^{2}\right\}, \\
p & =\xi^{2}+v^{2}{ }_{100}+\frac{v^{2}{ }_{110}}{3} .
\end{aligned}
$$

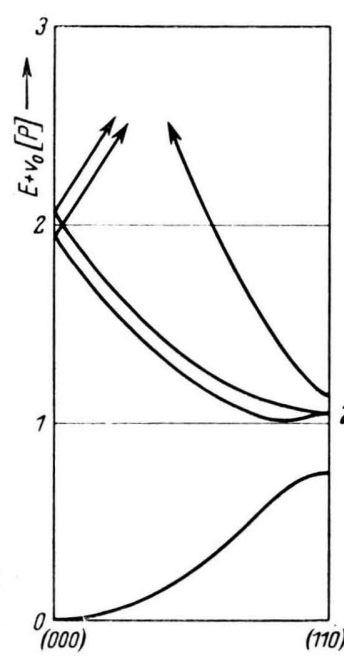

a
Man rechnet leicht nach, daß eine Entwicklung von (29) für kleine Werte von $v_{110}$ mit der Lösung (27) für $\eta=0$ übereinstimmt. Der Verlauf der Energiebänder über den Verbindungsgeraden $\overline{P_{0} P_{2}}$ bzw. $\overline{P_{2} P_{1}}$ kann mit Hilfe der Gln. (8), (26) und (27) berechnet werden und ist in Abb. $5 \mathrm{a}$ und $5 \mathrm{~b}$ dargestellt; bei $P_{0}=(000)$ ist dabei die Energieparabel des freien Elektrons verwendet worden.

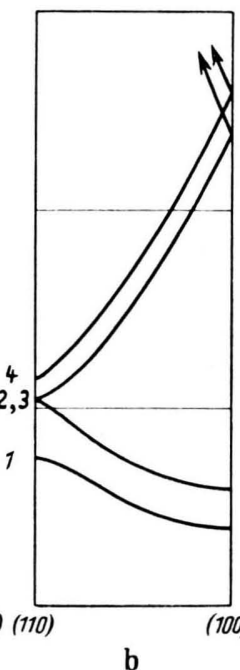

b

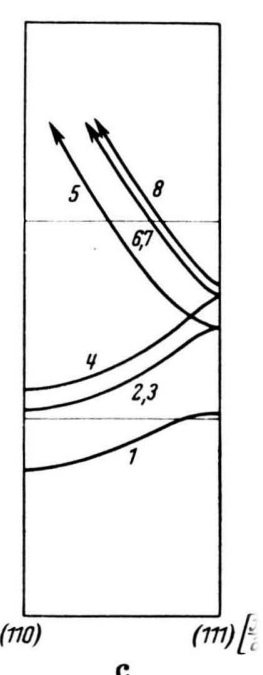

c
Abb. 5. Energiebänder bei Gitter $\Gamma_{c}$ über den Geraden $\overline{\mathrm{P}_{0} \mathrm{P}_{2}}, \overline{\mathrm{P}_{2} \mathrm{P}_{1}}$ und $\overline{\mathrm{P}_{2} \mathrm{P}_{3}} \cdot v_{100}=0,1 ; v_{100}: v_{110}: v_{111}=4: 2: 1$.

Die zu den verschiedenen Eigenwerten $\lambda_{l}$ gehörigen Lösungssysteme $\alpha_{l}^{r}$ von (19) sind durch die Zeilen der folgenden Matrix gegeben:

$$
\left(\alpha_{l}^{r}\right)=\left(\begin{array}{cccc}
1 & \frac{v_{x}}{2 \varepsilon_{x}} & \frac{v_{y}}{2 \varepsilon_{y}} & \frac{w}{2\left(\varepsilon_{x}+\varepsilon_{y}\right)} \\
\frac{v_{x} \delta}{\varepsilon_{y} N} & -\frac{2 \delta}{N} & \frac{w}{2 N} & \frac{v_{y} \delta}{\varepsilon_{y} N} \\
\frac{v_{y} \delta}{\varepsilon_{x} N} & -\frac{w}{2 N} & -\frac{2 \delta}{N} & \frac{v_{x} \delta}{\varepsilon_{x} N} \\
\frac{-w}{2\left(\varepsilon_{x}+\varepsilon_{y}\right)} & \frac{v_{y}}{2 \varepsilon_{y}} & \frac{v_{x}}{2 \varepsilon_{x}} & 1
\end{array}\right)+o\left(v_{110}^{2}\right)
$$

mit dem Normierungsfaktor

$N=\sqrt{4 \delta^{2}+\frac{w^{2}}{4}, 2 \delta \equiv \varepsilon_{x}-\varepsilon_{y} .}$

Die Beibehaltung des in $v_{110}$ quadratischen Terms $w^{2}$ ist notwendig, weil $\delta$ auf dem Geradenpaar $\Delta_{x}{ }^{2}=\Delta_{y}{ }^{2}$ verschwindet.

Wenn wir darauf verzichten, die Störung $\varphi$ der Eigenfunktionen mitzunehmen, lauten die neuen Eigenfunktionen 


$$
\bar{\chi}_{l}=\sum_{r=1}^{4} \alpha_{l}^{r} \chi_{r} \quad\left(\underset{r=1}{\stackrel{4}{N}}\left(\alpha_{l}^{r}\right)^{2}=1\right)
$$

2. Das räumliche kubische Gitter $\Gamma_{c}$

Um in der Umgebung der Stelle $P_{3}=(111)$ (vgl. Abb. 2) die Energiebänder näherungsweise berechnen zu können, fügen wir zu den bisher betrachteten Störungen

$$
\begin{gathered}
H^{3} \equiv-v_{111}\left(e^{2 i x}+e^{-2 i x}\right)\left(e^{2 i y}+e^{-2 i y}\right) \\
\left(e^{2 i z}+e^{-2 i z}\right)
\end{gathered}
$$

additiv hinzu. Folgende acht Wellen sind in dem positiven Oktanten der ersten B.-Zone näherungsweise entartet:

$\mathfrak{f}, \mathfrak{f}-2 \mathfrak{b}_{1}, \mathfrak{f}-2 \mathfrak{b}_{2}, \mathfrak{k}-2 \mathfrak{b}_{3}, \mathfrak{f}-2\left(\mathfrak{b}_{1}+\mathfrak{b}_{2}\right)$,

$\mathfrak{f}-2\left(\mathfrak{b}_{1}+\mathfrak{b}_{3}\right), \mathfrak{f}-2\left(\mathfrak{b}_{2}+\mathfrak{b}_{3}\right), \mathfrak{f}-2\left(\mathfrak{b}_{1}+\mathfrak{b}_{2}+\mathfrak{b}_{3}\right)$.

Den Vektor $\mathfrak{f}$ zerlegen wir wie früher gemä $ß \mathfrak{f}=\mathfrak{f}_{0}{ }^{(3)}$ $+\mathfrak{f}_{1}{ }^{(3)}\left(\mathfrak{f}_{0}{ }^{(3)}=(111), \mathfrak{f}_{1}^{(3)}=\left(\Delta_{x}, \Delta_{y}, \Delta_{z}\right)\right)$.

Die Störung $H^{0}$ und $H^{1}$ wird durch den in den drei Koordinaten separierbaren Ansatz berücksichtigt:

$$
\begin{array}{r}
\chi_{1}=\frac{1}{\sqrt{\pi^{3}}} \chi_{u}(x) \cdot \chi_{u}(y) \cdot \chi_{u}(z), \\
\chi_{2}=\frac{1}{\sqrt{\pi^{3}}} \chi_{0}(x) \cdot \chi_{u}(y) \cdot \chi_{u}(z), \\
\chi_{3}=\frac{1}{\sqrt{\pi^{3}}} \chi_{u}(x) \cdot \chi_{0}(y) \cdot \chi_{u}(z), \\
\chi_{4}=\frac{1}{\sqrt{\pi^{3}}} \chi_{0}(x) \cdot \chi_{0}(y) \cdot \chi_{u}(z), \\
\Delta_{1}=E_{1}-\bar{E}^{(3)}=-\varepsilon_{x}-\varepsilon_{y}-\varepsilon_{z}, \\
\Delta_{2}=E_{2}-\bar{E}^{(3)}=+\varepsilon_{x}-\varepsilon_{y}-\varepsilon_{z}, \\
\Delta_{3}=E_{3}-\bar{E}^{(3)}=-\varepsilon_{x}+\varepsilon_{y}-\varepsilon_{z}, \\
\Delta_{4}=E_{4}-\bar{E}^{(3)}=+\varepsilon_{x}+\varepsilon_{y}-\varepsilon_{z} .
\end{array}
$$

$\Delta_{r}$ sind wieder die Abweichungen von der mittleren Energie $\bar{E}^{(3)}$ der acht Funktionen; die fehlenden Funktionen $\chi_{5}$ bis $\chi_{8}$ erhält man aus $\chi_{1}$ bis $\chi_{4}$, indem $\operatorname{man} \chi_{u}(z)$ durch $\chi_{0}(z)$ ersetzt; in $\Delta_{1}$ bis $\Delta_{4}$ ist analog $+\varepsilon_{z}$ statt $-\varepsilon_{z}$ zu setzen. Mit einem (17) entsprechenden Ansatz bekommen wir die neuen Eigenfunktionen

$$
\bar{\chi}_{l}=\sum_{r=1}^{8} \alpha_{l}^{r} \chi_{r} \quad\left(\sum_{l=1}^{8}\left(\alpha_{l}^{r}\right)^{2}=1\right)
$$

aus dem linearen homogenen Gleichungssystem

$$
\begin{aligned}
& \stackrel{8}{r=1}_{r}\left[\left(\lambda-\Delta_{r}\right) \delta_{r m}-T_{r m}^{2}-T_{r m}^{3}\right] \alpha^{r}=0(m=1, \ldots, 8) \\
& \text { mit den Matrizen } \\
& \qquad T_{r m}^{2}=\left(\chi_{r}, H^{2} \chi_{m}\right), \quad T_{r m}^{3}=\left(\chi_{r}, H^{3} \chi_{m}\right) .
\end{aligned}
$$

Ihre wichtigsten Elemente sind

$$
\begin{aligned}
u^{x} \equiv-\left(\chi_{1}, V^{x} \chi_{1}\right)=v_{110} \frac{v^{2}{ }_{100}}{\varepsilon_{y} \varepsilon_{z}}, \\
U \equiv-\left(\chi_{1}, H^{3} \chi_{1}\right)=v_{111} \frac{v^{2}{ }_{100}}{\varepsilon_{x} \varepsilon_{y} \varepsilon_{z}}
\end{aligned}
$$

\begin{tabular}{|c|c|c|c|c|}
\hline$s$ & $\mathfrak{f}_{v}(s)\left\lceil\frac{\pi}{a}\right]$ & $\begin{array}{l}\text { Anzahl } \\
\text { der } \\
\text { Wellen }\end{array}$ & Aufspaltung & $\begin{array}{l}\text { Viel- } \\
\text { fach- } \\
\text { heit }\end{array}$ \\
\hline 1 & $(100)$ & 2 & $\pm v_{100} *$ & je 1 \\
\hline 2 & $(110)$ & 4 & 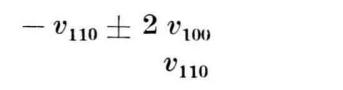 & $\begin{array}{c}\text { je } 1 \\
2\end{array}$ \\
\hline 3 & (111) & 8 & $\begin{array}{c}-3 v_{110} \pm\left(3 v_{100}+v_{111}\right) \\
v_{110} \pm\left(v_{100}-v_{111}\right)\end{array}$ & $\begin{array}{l}\text { je } 1 \\
\text { je } 3\end{array}$ \\
\hline 4 & $(200)$ & 6 & $\begin{array}{r}-v_{2 v 0}-4 v_{110} \\
-v_{200}+2 v_{110} \\
v_{200}\end{array}$ & $\begin{array}{l}1 \\
2 \\
3\end{array}$ \\
\hline 5 & $\begin{array}{l}\left(2 \frac{1}{2} \frac{1}{2}\right) \\
\left(\frac{3}{2} \frac{3}{2} 0\right)\end{array}$ & 3 & $\begin{array}{c}-v_{200} / 2 \pm V v_{200}^{2} / 4+2 v_{111}^{2} \\
v_{200}\end{array}$ & $\begin{array}{c}\text { je } 1 \\
1\end{array}$ \\
\hline 6 & $(210)$ & 4 & 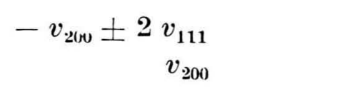 & $\begin{array}{c}\text { je } 1 \\
2\end{array}$ \\
\hline
\end{tabular}

$u^{y}$ und $u^{z}$ erhält man aus $u^{x}$, indem man zyklisch $x, y, z$ vertauscht. Für $\mathfrak{f}_{1}^{(3)}=0$ sind nur die Diagonalelemente von $T^{2}$ und $T^{3}$ von Null verschieden. Die näherungsweise Auflösung der Säkulargleichung des Gleichungssystems (36), welche quadratische Glieder in $v_{110}$ und $v_{111}$ vernachlässigt, liefert

$$
\begin{aligned}
& \lambda_{1}=-\varepsilon_{x}-\varepsilon_{y}-\varepsilon_{z}-u^{x}-u^{y}-u^{z}-U+\mathrm{o}\left(v^{2}{ }_{110}, v^{2}{ }_{111}\right) \\
& { }_{2}=+---+++ \\
& \text { 3 }=-+-+1+ \\
& \text { 4 }=++-+\infty- \\
& 5=-+++\infty+ \\
& { }_{6}=+-+\infty+
\end{aligned}
$$

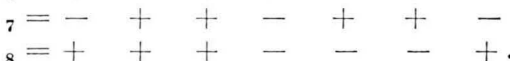

Für unsere Zwecke wird es genügen, das Koeffizien. tenschema der $\alpha^{r}$ in der Näherung

$$
\alpha_{l}^{r}=\delta_{l}^{r}+o\left(v_{110}, v_{111}\right) \quad(r, l=1, \ldots, 8)
$$

zu kennen. Der Energiebänderverlauf zwischen den Punkten $\mathrm{P}_{2}$ und $\mathrm{P}_{3}$ ist in Abb. $5 \mathrm{c}$ dargestellt; er ist

* Unter $v$ ist jeweils der Betrag des Fourier-Koeffizienten zu verstehen.

Tab. 1. Energieaufspaltung bei kubischen Gittern für die ersten sechs Resonanzstellen. 
aus (26) und (39) zu entnehmen. Tab. 1 gibt neben der Aufspaltung in $\mathfrak{f}_{0}^{(1)}=(100)$ [vgl. Gl. (8)] und $\mathfrak{f}_{0}^{(2)}$ [etwa aus Gl. (26)] u. a. die aus (39) zu entnehmende Aufspaltung in $\mathfrak{f}_{0}{ }^{(3)}$.

\section{Das flächenzentrierte kubische Gitter $\Gamma^{\prime}{ }_{c}$}

Die erste B.-Zone des Gitters $\Gamma^{\prime}{ }_{c}$ wird begrenzt durch die Flächen vom Typ $\{200\}$ und $\{111\}$ (Abb.6). Dagegen berührt die nächst höhere Reflexionsebene vom Typ $\{220\}$ nicht mehr die erste Zone. Neben der Störung $H^{3}$ ist somit die Störung $H^{4}$

$$
\begin{aligned}
& H^{4}=V_{2 x}+V_{2 y}+V_{2 z}, \\
& \quad V_{2 x_{l}}=-v_{200}\left(e^{4 i x_{l}}+e^{-4 i x_{l}}\right)
\end{aligned}
$$

für das Verhalten des Elektrons in erster Linie verantwortlich. Weiter ist anzumerken, daß theoretisch beim kristallographischen Bl-Typ (NaCl-Typ) stets $\left|v_{200}\right|>\left|v_{111}\right|$ ist. Für einen Vektor $\mathfrak{f}$ im positiven Oktanten der ersten B.-Zone wird im allgemeinen eine 14-fache Entartung näherungsweise eintreten, d. h. es wird ein 15-facher Funktionenansatz notwendig sein mit ebenen Wellen, welche zu folgenden Wellenzahlvektoren gehören:

[1] $\mathfrak{f},[2] \mathfrak{f}-4 \mathfrak{b}_{1},[3] \mathfrak{f}-4 \mathfrak{b}_{2},[4] \mathfrak{f}-4 \mathfrak{b}_{3}$,

$[5] \mathfrak{f}-4\left(\mathfrak{b}_{1}+\mathfrak{b}_{2}\right),[6] \mathfrak{f}-4\left(\mathfrak{b}_{1}+\mathfrak{b}_{3}\right)$,

$[7] \mathfrak{f}-4\left(\mathfrak{b}_{2}+\mathfrak{b}_{3}\right),[8] \mathfrak{f}-4\left(\mathfrak{b}_{1}+\mathfrak{b}_{2}+\mathfrak{b}_{3}\right)$,

$[9] \mathfrak{f}-2\left(\mathfrak{b}_{1}+\mathfrak{b}_{2}+\mathfrak{b}_{3}\right),[10] \mathfrak{f}-2\left(\mathfrak{b}_{1}-\mathfrak{b}_{2}+\mathfrak{b}_{3}\right)$, $[11] \mathfrak{f}-2\left(\mathfrak{b}_{1}+\mathfrak{b}_{2}-\mathfrak{b}_{3}\right),[12] \mathfrak{f}-2\left(\mathfrak{b}_{1}-\mathfrak{b}_{2}-\mathfrak{b}_{3}\right)$, $[13] \mathfrak{f}+2\left(\mathfrak{b}_{1}+\mathfrak{b}_{2}-\mathfrak{b}_{3}\right),[14] \mathfrak{f}+2\left(\mathfrak{b}_{1}-\mathfrak{b}_{2}+\mathfrak{b}_{3}\right)$, $[15] \mathfrak{f}+2\left(\mathfrak{b}_{1}-\mathfrak{b}_{2}-\mathfrak{b}_{3}\right)$.

Im allgemeinen ist nur ein Teil dieser Wellen von Bedeutung; es müssen aber in jedem Falle in der Umgebung einer Resonanzstelle $\mathfrak{f}_{0}$ diejenigen Wellen berücksichtigt werden, welche in $\mathfrak{f}_{0}$ vollständig entartet sind. Um die Energieaufspaltung in den Punkten $\mathrm{P}_{3}, \mathrm{P}_{4}, \mathrm{P}_{5}$ und $\mathrm{P}_{6}$ (vgl. Abb. 6) zu berechnen und auf den zugehörigen Verbindungsgeraden zu ermitteln, verwenden wir folgende vier Ansätze.

Ansatz $1 \mathrm{im} \mathrm{Punkt} \mathrm{P}_{3}=$ (111). In $\mathrm{P}_{3}$ sind die Wellen [1] und [9] vollständig entartet und durch die $H^{3}$-Störung wie folgt verbunden

$$
\begin{gathered}
\chi_{2}^{1}=\frac{1}{\sqrt{\pi^{3}}} \chi_{0}^{u}(\sqrt{3} s), \Delta_{\frac{1}{2}}=E_{\frac{1}{2}}-\bar{E}^{(3)}=\mp \varepsilon_{\sqrt{3} s} \\
s \equiv(x+y+z) / \sqrt{3} .
\end{gathered}
$$

Das Ergebnis der Störungsrechnung ist dasselbe wie in der Einleitung, es ist lediglich in (8) bzw. (10) $m=\sqrt{3} \mathrm{zu}$ wählen und $x$ durch $s$ zu ersetzen.

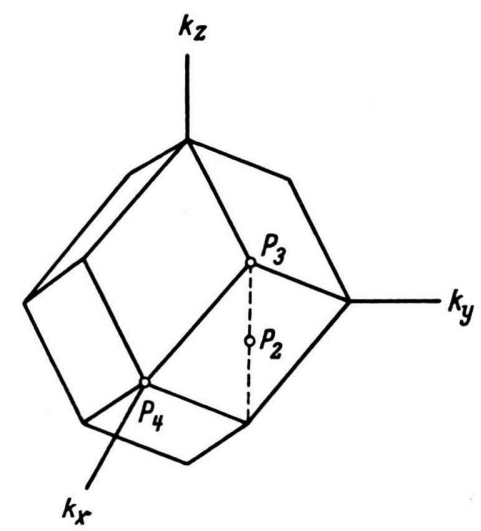

Abb. 6. Erste B.-Zone des Gitters $\Gamma_{c}{ }^{\prime}$ mit den Punkten $\mathrm{P}_{3}=(111), \mathrm{P}_{4}=(200), \mathrm{P}_{5}=\left(2 \frac{1}{2} \frac{1}{2}\right), \mathrm{P}_{5}{ }^{\prime}=\left(0 \frac{3}{2} \frac{3}{2}\right)$, $\mathrm{P}_{6}=(210)$.

Bei Ansatz 2, der zum Punkt $\mathrm{P}_{4}=(200)$ gehört, sind entsprechend die Wellen [1] und [2], jetzt aber durch die Störung $H^{4}$ gekoppelt

$$
\chi_{2}^{1}=\frac{1}{\sqrt{\pi^{3}}} \chi_{0}^{u}(2 \mathrm{x}), \Delta_{2}=E_{1}-\bar{E}^{(4)}=\mp \varepsilon_{2 x} .
$$

Die Störung $H^{3}$ liefert, auf die Funktionen (43) angewendet, keine weitere Aufspaltung, vielmehr müssen die vier Wellen [9], [10], [11] und [12] noch hinzugefügt werden, welche ihrerseits durch die Störung $H^{4}$ wie folgt verbunden sind:

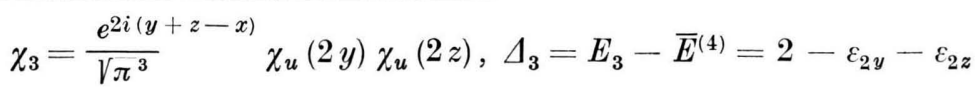

$$
\begin{aligned}
& \begin{array}{lllllll}
{ }_{4}= & u & 0 & { }_{4}= & 4 & = & - \\
{ }_{5}= & 0 & u & { }_{5}= & 5 & = & + \\
{ }_{6}= & 0 & 0 & { }_{6}= & { }_{6} & = & +
\end{array}
\end{aligned}
$$

Die Störung $H^{3}$ verbindet nun die Funktionen (43) und (44) zu einem gemeinsamen Ansatz

$$
\bar{\chi}_{l}=\sum_{r=1}^{6} \alpha_{l}{ }^{r} \chi_{r} \quad\left(\sum_{l=1}^{6}\left(\alpha_{l}{ }^{r}\right)^{2}=1\right),
$$


worin die Koeffizienten aus einem Gleichungssystem sechsten Grades

$$
\sum_{r=1}^{6}\left[\left(\lambda-\Delta_{r}\right) \delta_{r m}-T_{r m}^{3}\right] \alpha^{r}=0 \quad(m=1, \ldots, 6)
$$

zu entnehmen sind. Darin ist

$$
\left(T_{r m}^{3}\right) \equiv\left(\chi_{r}, H^{3} \chi_{m}\right)=-\left(\begin{array}{lllccc}
0 & 0 & \boldsymbol{\tau}_{+++} & \boldsymbol{\tau}_{++-} & \boldsymbol{\tau}_{+-+} & \boldsymbol{\tau}_{+--} \\
0 & 0 & \boldsymbol{\tau}_{-++} & \boldsymbol{\tau}_{-+-} & \boldsymbol{\tau}_{--+} & \boldsymbol{\tau}_{---} \\
\boldsymbol{\tau}_{+++} & \boldsymbol{\tau}_{-++} & 0 & 0 & 0 & 0 \\
\boldsymbol{\tau}_{++-} & \boldsymbol{\tau}_{-+-} & 0 & 0 & 0 & 0 \\
\tau_{+-+} & \boldsymbol{\tau}_{--+} & 0 & 0 & 0 & 0 \\
\tau_{+--} & \tau_{---} & 0 & 0 & 0 & 0
\end{array}\right)
$$

mit den Elementen

$\tau_{ \pm \pm \pm}=v_{111} \sqrt{\left(1 \pm \frac{v_{200}}{\varepsilon_{2 x}}\right)\left(1 \pm \frac{v_{200}}{\varepsilon_{2 y}}\right)\left(1 \pm \frac{v_{200}}{\varepsilon_{2 z}}\right)}$.

Die Lösung der Säkulargleichung von (46) ist für die $\{200\}$-Ebene, also für $\Delta_{x}=0\left(\tau_{- \pm \pm}=0\right)$ gut angebbar, da nur das dritte Niveau mit dem ersten und zweiten bei $\Delta_{y}=\Delta_{z}=0$ gekoppelt ist, während das vierte bis sechste Niveau wegen des großen Abstandes von den ersten Bändern als ungekoppelt angesehen werden können. Es ist

$$
\begin{aligned}
& \lambda_{3}=\frac{\Delta_{3}}{2}-\frac{v_{200}}{2} \mp \sqrt{\left(\frac{\Delta_{3}}{2}+\frac{v_{2(0)}}{2}\right)^{2}+\tau_{+++}^{2}} \\
& \lambda_{2}=\Delta_{2}=v_{200} \\
& \lambda_{4} \approx \Delta_{4}, \quad \lambda_{5} \approx \Delta_{5}, \quad \lambda_{6} \approx \Delta_{6} .
\end{aligned}
$$

Den Energieverlauf in der Umgebung des Punktes $P_{5}=\left(2 \frac{1}{2} \frac{1}{2}\right)$ können wir entweder aus Ansatz 2 mit der Transformation

$$
\Delta_{y}=\frac{1}{2}+\Delta_{y}^{(5)}, \Delta_{z}=\frac{1}{2}+\Delta_{z}^{(5)}
$$

gewinnen, oder wir berechnen Eigenfunktionen und Energieverlauf in einem Ansatz 3 analog zu oben neu, indem wir lediglich die in $\mathfrak{f}_{0}^{(5)}$ exakt entarteten Wellen [1], [2] und [9] berücksichtigen. Die Abweichung von der mittleren Energie $\bar{E}^{(5)}$ in der Ebene $\Delta_{x}=0$ lautet dann:

$$
\begin{aligned}
& \lambda_{3}=-\frac{\Delta_{y}^{(5)}+\Delta_{z}^{(5)}}{2}-\frac{v_{200}}{2} \\
& \mp \sqrt{\left(\Delta_{y}^{(5)}+\Delta_{z}^{(5)}-\frac{v_{200}}{2}\right)^{2}+2 v_{111}^{2}}
\end{aligned}
$$

$\lambda_{2}=v_{200}+\frac{1}{2}\left(\Delta_{y}^{(5)}+\Delta_{z}^{(5)}\right)$.

Man rechnet leicht nach, daß in $\mathrm{P}_{5}^{\prime}=\left(\frac{3}{2} \frac{3}{2} 0\right)$ die gleiche Aufspaltung (50) vorliegt, wenn man die Wellen [1], [9] und [10] zugrunde legt.
Weiter können wir die Energie in der Umgebung des Punktes $\mathrm{P}_{6}=(210)$ entweder so berechnen, daß wir in Ansatz 2 die Größe $\Delta_{y}=1+\Delta_{y}^{(6)}$ setzen, oder daß wir in einem Ansatz 4 die vier in $\mathfrak{f}_{0}^{\left({ }^{(6)}\right.}$ voll entarteten Wellen [1], [2], [8] und [10] den Störungen $H^{4}$ und $H^{3}$ successive unterwerfen. Für zwei Fälle soll die Abweichung von der mittleren Energie $\bar{E}^{(6)}$ mitgeteilt werden, wenn man den letzteren Weg beschreitet. Erstens folgt für $\Delta_{x}=\Delta_{z}=0$

$$
\begin{aligned}
& \lambda_{\frac{1}{2}}=-v_{200} \mp \sqrt{\Delta_{y}^{(6) 2}+4 v_{111}^{2}} \\
& \lambda_{4}^{3}=v_{200} \pm \Delta_{y}^{(6)},
\end{aligned}
$$

dann für $\Delta_{y}^{(6)}=0, \Delta_{x}=\Delta_{z}$

$$
\begin{aligned}
& \lambda_{1}=\mp v_{111}-\sqrt{4 \Delta_{x}^{2}+\left(v_{200} \pm v_{111}\right)^{2}} \\
& \lambda_{4}^{3}=\mp v_{111}+\sqrt{4 \Delta_{x}^{2}+\left(v_{200} \pm v_{111}\right)^{2}} .
\end{aligned}
$$

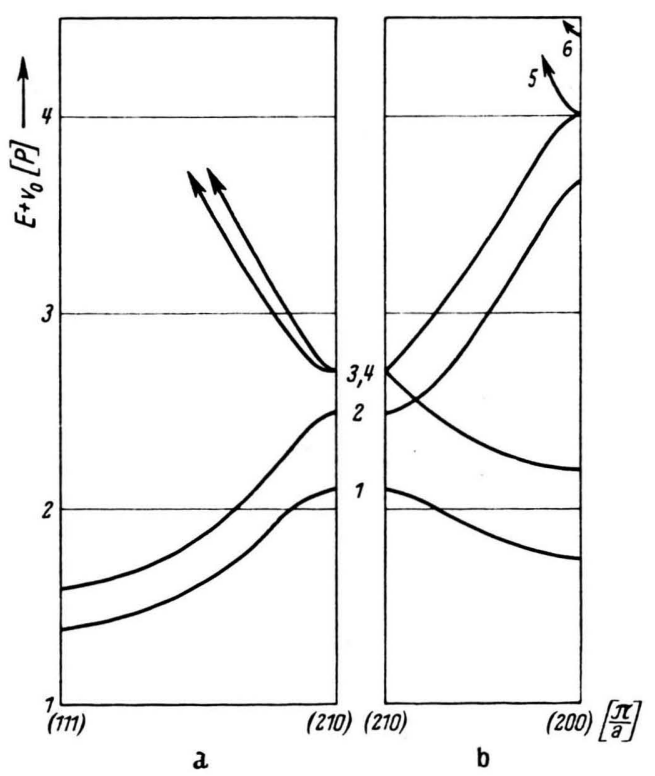

Abb. 7. Energiebänder beim Gitter $\Gamma_{c}{ }^{\prime}$ über den Geraden $\overline{\mathrm{P}_{3} \mathrm{P}_{6}}$ und $\overline{\mathrm{P}_{6} \mathrm{P}_{4}} \cdot v_{200}=0,2 ; v_{200}: v_{111}=2: 1$. 
In Abb. 7 ist der Verlauf der Energiebänder auf den Geraden $\overline{\mathrm{P}_{3} \mathrm{P}_{6}}$ und $\overline{\mathrm{P}_{6} \mathrm{P}_{4}}$ angegeben, wie er sich aus den Gln. (43), (49), (51) und (52) berechnen läßt. Speziell ist die Energieaufspaltung in $\mathfrak{f}_{0}{ }^{(5)}$ und $\mathfrak{f}_{0}{ }^{(6)}$ in Tab. 1 aufgeführt, wie sie sich aus Ansatz 3 und 4 ergibt.

\section{Das raumzentrierte kubische Gitter $\Gamma_{c}^{\prime \prime}$}

In Abb. 8 ist die erste B.-Zone des Gitters $\Gamma_{c}{ }^{\prime \prime}$ dargestellt, welche durch die B.-Ebenen vom Typ $\{110\}$ begrenzt wird. Die Ebenen vom Typ $\{200\}$ berühren die Zone, während die nächst höheren Ebenen vom Typ $\{211\}$ höhere Zonen einschließen.

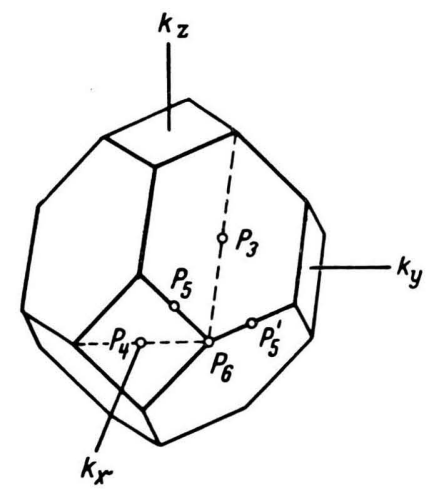

Abb. 8. Erste B.-Zone des Gitters $\Gamma_{c}{ }^{\prime \prime}$ mit den Punkten $P_{2}=(110), P_{3}=(111)$ und $P_{4}=(200)$.

Es werden darum die Störungen $H^{2}$ und $H^{4}$ für das Verhalten eines Elektrons in der ersten B.-Zone charakteristisch sein. Im allgemeinen wird für einen Wellenzahlvektor im positiven Oktanten der ersten Zone näherungsweise eine 16 -fache Entartung vorliegen, d. h. wir werden mit einem 17 -fachen Funktionenansatz rechnen müssen, nämlich mit den Wellen [1] - [8] aus Abschnitt 3 und mit den Wellen der Wellenzahlen

$$
\begin{aligned}
& {[9] \mathfrak{f}-2\left(\mathfrak{b}_{1}+\mathfrak{b}_{2}\right),[10] \mathfrak{f}-2\left(\mathfrak{b}_{1}+\mathfrak{b}_{3}\right),} \\
& {[11] \mathfrak{f}-2\left(\mathfrak{b}_{2}+\mathfrak{b}_{3}\right),[12] \mathfrak{f}-2\left(\mathfrak{b}_{1}-\mathfrak{b}_{2}\right),} \\
& {[13] \mathfrak{f}-2\left(\mathfrak{b}_{2}-\mathfrak{b}_{1}\right),[14] \mathfrak{f}-2\left(\mathfrak{b}_{1}-\mathfrak{b}_{3}\right),} \\
& {[15] \mathfrak{f}-2\left(\mathfrak{b}_{3}-\mathfrak{b}_{1}\right),[16] \mathfrak{f}-2\left(\mathfrak{b}_{2}-\mathfrak{b}_{3}\right),} \\
& {[17] \mathfrak{f}-2\left(\mathfrak{b}_{3}-\mathfrak{b}_{2}\right) .}
\end{aligned}
$$

Wiederum werden wir in bestimmten Gebieten der Zone mit weniger Wellen auszukommen versuchen.

Im Ansatz 1 berechnen wir den Einfluß der $H^{2}$ Störung des Punktes $\mathrm{P}_{2}=(110)$ auf die dort exakt

entarteten Eigenfunktionen [1] und [9] wie in der Einleitung. Es ist

$$
\begin{gathered}
\chi_{2}^{1}=\frac{1}{\sqrt{\pi^{3}}} \chi_{0}^{u}(\sqrt{2} t), \Delta_{\frac{1}{2}}=E_{\frac{1}{2}}-\bar{E}^{(2)}=\mp \varepsilon_{\sqrt{2}} t \\
\sqrt{2} t=x+y
\end{gathered}
$$

Im Punkt $\mathrm{P}_{3}=(111)$ sind die Wellen [1], [9], [10] und [11] entartet. Ein linearer Ansatz 2 dieser vier Wellen führt bei Berücksichtigung der Störung $\mathrm{H}^{2}$ auf ein homogenes Gleichungssystem vierten Grades in den Koppelungsparametern, dessen Säkulargleichung auf der Geraden $\Delta_{x}^{(3)}=\Delta_{y}^{(3)}=0$ die Lösung hat:

$$
\begin{aligned}
& \lambda_{\frac{1}{2}}=-v_{110} \mp \sqrt{\Delta_{z}^{(3) 2}+4 v_{110}^{2}} \\
& \lambda_{4}^{3}=v_{110} \pm \Delta_{z}^{(3)} .
\end{aligned}
$$

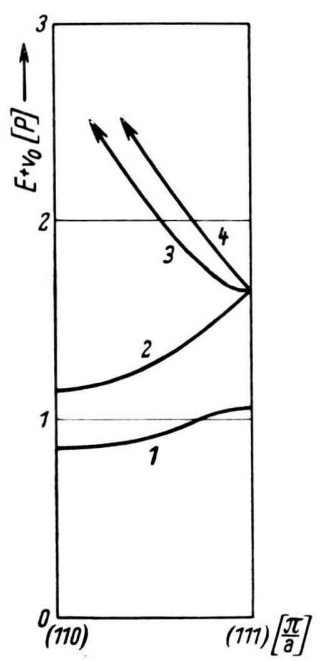

Abb. 9. Energiebänder beim Gitter $\Gamma_{c}{ }^{\prime \prime}$ über der Geraden $\overline{\mathrm{P}_{2} \mathrm{P}_{3}}$. $v_{110}=0,15$.
An der Stelle $\mathrm{P}_{4}=(200)$ sind die Wellen [1], [2], [9], [10], [12] und [14] entartet. Einen linearen Ansatz 3 dieser sechs Wellen können wir wie in Abschnitt 3 dazu benutzen, zunächst Störung $\mathrm{H}^{4}$, dann $\mathrm{H}^{3} \mathrm{zu}$ berücksichtigen. Die allgemeine Lösung dieses Ansatzes soll nicht weiter verfolgt werden. Die Aufspaltung in $\mathfrak{f}_{0}{ }^{(4)}$ selbst ist in Tab. 1 vermerkt, während die in die sem Abschnitt gefundenen Aufspaltungen in $\mathfrak{f}_{0}^{(2)}$ und $\mathfrak{f}_{0}{ }^{(3)}$ als Spezialfälle beim Gitter $\Gamma_{c}$ schon gewonnen wurden. In Abb. 9 sind die Energiebänder über der Geraden $\overline{\mathrm{P}_{2} \mathrm{P}_{3}}$ aufgetragen, wie sie sich nach den Gln. (53) und (54) berechnen lassen.

\section{Folgerung}

Als Konsequenz aus den in Tab. 1 zusammengestellten Energieaufspaltungen ergibt sich die Möglichkeit, die Energielücke zwischen erstem und zweitem Band für die drei diskutierten Gitter als Funktion der wichtigsten Fourierkoeffizienten auszudrücken. Dies ist in Tab. 2 geschehen. Diese zeigt zunächst, daß die für ein Isolatormodell maßgebliche Energielücke beim Gitter $\Gamma_{c}$ in der Ecke stets größer ist als auf den Kanten und Flächenmitten 
der würfelförmigen ersten B.-Zone, falls die Koeffizienten $v_{110}$ und $v_{111}$ von Null verschieden sind. Das bedeutet, daß die Energielücke in der Folge der Richtungen [100] - [110] - [111] ansteigt. Für das Gitter $\Gamma_{c}^{\prime \prime}$ folgt, daß stets in der Mitte der die erste B.-Zone begrenzenden $\{110\}$-Flächen, also in der [110]-Richtung, die geringste Energielücke zu finden ist, während die Aufspaltung nach den Ecken zunimmt, und zwar in der Folge [111] - [100]. Dagegen läßt sich für das $\Gamma_{c}{ }^{\prime}$-Gitter wegen des im Ein- zelfall erst zu ermittelnden Größenverhältnisses $\delta=\left|v_{200} / v_{111}\right|$ keine so einfache Aussage machen. Ist $\delta=1$, so liegt in der [111]- und [100]-Richtung die gleiche minimale Energielücke vor, welche in Richtung $[110]-[210]$ ansteigt.

Herrn Prof. Dr. W. Franz sei an dieser Stelle herzlich gedankt für die Anregung zur Dissertation über die Theorie der inneren Feldemission in isolierenden Einkristallen und für seine fördernde Anteilnahme bei der Durchführung der Arbeit.

\begin{tabular}{|c|c|c|c|c|}
\hline Gitter & $\mathfrak{f}_{0}\left\lceil\frac{\pi}{a}\right\rceil$ & Richtung & Energielücke & $\begin{array}{l}\text { Anzahl } \\
\text { der } \\
\text { Wellen }\end{array}$ \\
\hline$\Gamma_{c}$ & $\begin{array}{l}(100) \\
(110) \\
(111)\end{array}$ & $\begin{array}{l}{[100]} \\
{[110]} \\
{[111]}\end{array}$ & $\begin{array}{l}2 v_{100} * \\
2 \operatorname{Min}\left[v_{106}+v_{110} ; 2 v_{100}\right] \\
2 \operatorname{Min}\left[3 v_{100}+v_{111} ; v_{100}+2 v_{110}+v_{111} ; 2 v_{100}+2 v_{111}\right]\end{array}$ & $\begin{array}{l}2 \\
4 \\
8\end{array}$ \\
\hline$\Gamma_{c}^{\prime}$ & $\begin{array}{l}(111) \\
(200) \\
\left(\frac{3}{2} \frac{3}{2} 0\right) \\
\left(2 \frac{1}{2} \frac{1}{2}\right) \\
(210)\end{array}$ & $\begin{array}{l}{[111]} \\
{[100]} \\
{[110]} \\
{[411]} \\
{[210]}\end{array}$ & $\begin{array}{l}2 v_{111} \\
2 v_{200} \\
\operatorname{Min}\left[\sqrt{v_{200}^{2}+8 v_{111}^{2}} ; \frac{3}{2} v_{200}+\frac{1}{2} \sqrt{v_{200}^{2}+8 v_{111}^{2}}\right] \\
2 \operatorname{Min}\left[2 v_{111} ; v_{200}+v_{111}\right]\end{array}$ & $\begin{array}{l}2 \\
2 \\
3 \\
4\end{array}$ \\
\hline$\Gamma_{c}^{\prime \prime}$ & $\begin{array}{l}(110) \\
(111) \\
(200)\end{array}$ & $\begin{array}{l}{[110]} \\
{[111]} \\
{[100]}\end{array}$ & $\begin{array}{l}2 v_{110} \\
4 v_{110} \\
2 \text { Min }\left[2 v_{110}+v_{200} ; 3 v_{110}\right]\end{array}$ & $\begin{array}{l}2 \\
4 \\
6\end{array}$ \\
\hline
\end{tabular}

* s. Fußnote unter Tab. 1.

Tab. 2. Energielücke zwischen erstem und zweitem Energieband. 